\title{
The role and impact of polyethylene glycol on anaphylactic reactions to COVID-19 nano-vaccines
}

\author{
Polyethylene glycol, used as a stabilizer in nanomedicine formulations, has recently been indicated as the possible \\ cause of the anaphylactic reactions against the COVID-19 mRNA-based vaccines, but the evidence supporting this \\ is contradictory, and other factors might be involved.
}

\author{
P. Bigini, M. Gobbi, M. Bonati, A. Clavenna, M. Zucchetti, S. Garattini and G. Pasut
}

T he approval by national and international drug agencies of the messenger RNA (mRNA)-based vaccines for COVID-19 has paved the way to the clinical application of solid lipid nanoparticles (SLNPs). For both products, phase-III clinical trials demonstrated great efficacy and safety, with scarce severe adverse events (SAEs), balanced across vaccine and placebo groups. Nevertheless, 4.7 and 2.8 cases per million of anaphylactic reactions have been registered during the first months of the vaccination campaign for the Pfizer/BioNTech ${ }^{1}$ and the Moderna vaccine, respectively ${ }^{2}$. Although these numbers are only slightly higher than the rates reported for influenza vaccines (1.3 per million doses $)^{3}$, perception of the risk has been greatly amplified by the extensive programme of recruitment, which has seen hundreds of millions of people worldwide undertake the vaccine since the vaccination campaign began. Moreover, the echo from newspapers, television and social media has abnormally distorted the encouraging messages published by the scientific community about the efficacy and safety of the vaccines.

Polyethylene glycol (PEG), the polymer used in these vaccines for coating the SLNPs, has been indicated as the principal suspect for these anaphylactic reactions ${ }^{4,5}$. PEG has a broad range of applications in many other products ${ }^{6}$, such as cosmetics, foods and pharmaceutical preparations. Its activity is crucial to maintain the colloidal stability of nanoparticles in biological fluids and to reduce their uptake by filter organs, improving their efficacy and safety after inoculation.

For a long time, PEG was classified as a non-immunogenic polymer, even when administered with Freund's adjuvant $^{7}$. Thanks to this inertness, it has been specifically adopted to reduce the immunogenicity of some biotech drugs, with very limited cases of anaphylaxis in subjects later found to be hypersensitive to PEG by skin prick tests ${ }^{8}$. The evidence that approximately one-third of those who experienced anaphylaxis after vaccination had previous history of allergic or anaphylactic reactions suggests that anti-PEG antibodies formed after exposure to PEGylated compounds may play a role? Indeed, recent research on plasma samples from hospitals or service companies based in the United States found that $65-76 \%$ of the general population had anti-PEG antibodies ${ }^{10,11}$ at an average concentration of $52 \mathrm{ng} \mathrm{ml}^{-1}$ (ref. ${ }^{10}$ ). However, in another multicentric study recruiting more than 1,300 subjects, the numbers were much lower, with $23-24 \%$ of individuals positive for either immunoglobulin M (IgM) or IgG able to recognize PEG, but among these only $4-9 \%$ had antibodies with a confirmed specificity for PEG (titres $\geq 80)^{12}$. This latter investigation agrees with other previous studies $^{13,14}$. Such controversial results probably stem from a difference in both the cohort recruitment and the analytical methods used for antibody detection. To shed light on this matter, protocols (recruitment, stratification, statistical analyses and experimental procedures) for the determination of anti-PEG antibodies should be harmonized.

It has been widely demonstrated that PEG may enhance its immunogenic response when conjugated with other entities such as proteins, liposomes or other types of nanoparticles ${ }^{7}$. More specifically, the conjugation of PEG to nanoparticles may lead to hypersensitivity reactions mainly involving the activation of the complement system, through a mechanism called complement activation-related pseudoallergy (CARPA). In the case of COVID-19 vaccines, CARPA can be activated even if the pre-existing anti-PEG antibodies have low affinity for PEG. In fact, since multiple PEG molecules cover the SLNPs surface, an early and weak anti-PEG/

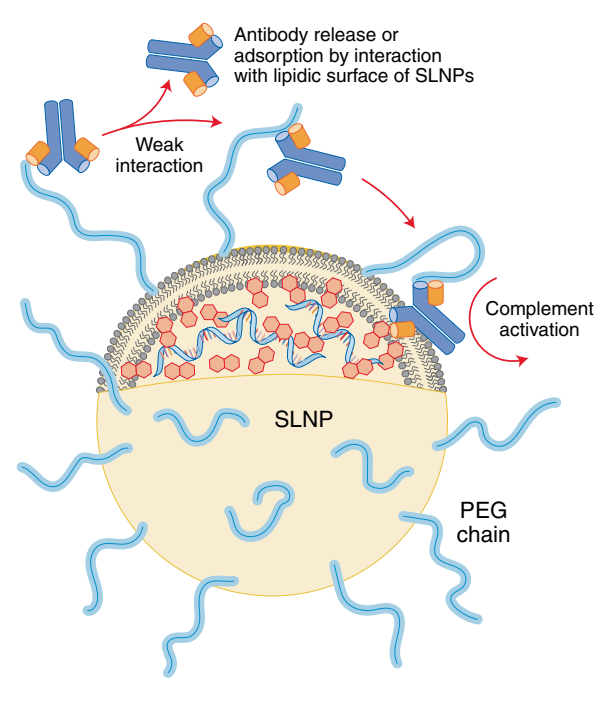

Lipid

Fig. 1 | Schematic representation of potential adsorption of anti-PEG antibodies on the surface of SLNPs. In SLNPs covered by multiple PEG molecules, absorption of PEG antibodies to the lipid surface might stabilise weak anti-PEG-PEG binding and activate the complement response.

PEG interaction can be stabilized by the adsorption of the antibody on the lipid moiety of SLNPs, thus triggering in turn the complement activation in a subset of very sensitive individuals (Fig. 1). Accordingly, SLNPs may increase the risk associated with anti-PEG antibodies compared with unbound PEGs ${ }^{15}$.

To better understand the role of anti-PEG antibodies associated with nanoparticles, it is worth looking at the data collected for Doxil, a PEGylated liposome currently used in the clinic for cancer therapy ${ }^{16}$. An in vitro study using mouse plasma demonstrated that pre-incubation of Doxil with commercial anti-PEG IgM at a concentration of $10 \mu \mathrm{g} \mathrm{ml}^{-1}$ activated 
the complement, assessed by the increase of C3a level, whereas incubation with anti-PEG IgG did not induce the activation of complement. However, the concentration of monoclonal commercial antibodies used in the study does not reflect the in vivo situation, where the average of anti-PEG antibodies in positive subjects was $52 \mathrm{ng}$ $\mathrm{ml}^{-1}$ (ref. ${ }^{10}$ ). In fact, the same study, when directly analysing the plasma of human volunteers, found no correlation between the concentration of pre-existing anti-PEG antibodies detected in the plasma samples by the authors, and the complement activation upon incubation with Doxil ${ }^{17}$. Therefore, the authors stated that the presence of pre-existing polyclonal antibodies reactive against PEG, methoxy-PEG or PEGylated liposomes may not accurately predict the complement activation by Doxil in vitro. Although with the bias of a limited sample size $(n=20)$, the study also showed that among the individuals with pre-existing anti-PEG IgM and IgG, there was a prevalence of women (about $80 \%$ ). Very interestingly, this percentage rose to close to $100 \%$ when higher antibody titres were considered $^{17}$.

Like Doxil, the SLNPs constituting Pfizer/BioNTech and Moderna vaccines bear a PEGylated surface that can interact with circulating antibodies and serum components. One could therefore correlate the almost exclusive occurrence of anaphylactic reactions following administration of the Pfizer/BioNTech and Moderna vaccines in women (63 out of 66 total cases) ${ }^{9}$ with the female prevalence of anti-PEG antibodies. However, a previous cohort study with a large sample size $(n=1,020)$ had reported that anti-PEG antibodies were present in $65 \%$ of females versus $35 \%$ of males, a smaller difference than found in ref. ${ }^{17}$, shedding doubts on whether the presence of anti-PEG antibodies can alone account for the high female/ male ratio of severe allergic reactions to the COVID-19 vaccines. Moreover, one must note that anaphylactic reactions - to drugs, foods or insect venoms - are generally more frequent among females, with about a 60:40 proportion ${ }^{18}$. Another piece of evidence that seems to exclude PEG as a key factor in SAEs is the persistence of female prevalence in the SAEs even in the cluster of population receiving non-PEGylated anti COVID-19 vaccines.

In contrast to many other viral forms, an imbalance between the sexes seems to be a peculiarity of the 'COVID story'. Whereas both the clinical severity and the mortality rate are significantly higher in males than in females ${ }^{19}$, the data on the prevalence of post-vaccination side effects show the opposite trend. The reasons why females are more reactive than males to the vaccines may be multiple and interconnected. Hormones and cell-mediated immune activation can play a great role either in reducing the virus propagation, in the case of infection, or in amplifying side-effects after vaccination. In line with the above functional hypothesis, a clear age-related trend exists in the female population, with women under 50 years old having a better prognosis after COVID infection but a higher frequency of side effects after vaccination. In addition, the mechanisms of how SLNPs interact with host cells could have a role in the difference of reactivity for each sex. Interestingly, a recent study showed that the intracellular rate of internalization of quantum dots can be influenced by cell developmental stage/age (amniotic stem cells versus differentiated cells from adults) and by cell sex. This evidence, albeit preliminary, may suggest an alternative mechanism for a sex/age-related response ${ }^{20}$.

A recent study correlated an episode of anaphylactic reaction after Pfizer/BioNTech vaccine in a woman with the presence of anti-PEG antibodies, determined by a skin prick test. However, the study also showed that three other subjects experiencing a similar systemic adverse reaction did not present pre-existing anti-PEG antibodies ${ }^{21}$. Overall, despite the limited number of cases described, the results of this study suggest that the role of PEG in the occurrence of anaphylactic reactions might be marginal.

The interaction between nanoparticles and the biological matrices that they encounter has been a matter of debate in the past decade, and many efforts have been made to characterize the 'bio-nano interaction'. It is clear that physico-chemical properties, such as type of material, stiffness, zeta-potential and surface coating, can affect the composition of the nanoparticle protein corona, and hence the nanoparticles' colloidal stability and their propensity to aggregate or to penetrate inside the cells after parenteral injection. Interactions of the nanoparticles with their environment are thought to occur mainly when they are systemically administered in the body. However, we cannot exclude upstream potential interactions, such as those occurring between the SLNPs and the syringes used for injection. For example, the SLNPs can interact with the sub-micrometre silicone droplets used as lubricant for the plunger, leading to disassembly and/ or aggregation of the SLNPs. Although this phenomenon is well known to occur in the case of biotech drugs ${ }^{22,23}$, a recent study demonstrated the stability of Pfizer/
BioNTech vaccine in these syringes under 'real-life' use, while only strong mechanical stress, such as shaking, can promote SLNP aggregation and mRNA degradation ${ }^{24}$.

In conclusion, although anaphylaxis occurs rarely after vaccination, this is a life-threatening allergic reaction that demands the development of safer drug delivery systems, vaccines and bio-inspired stabilizing agents. On the other hand, PEG has traditionally been used to avoid SLNP aggregation and improve their biocompatibility, and its use, supported by an established clinical database of PEGylated products, would mitigate the risks of developing new stabilizing materials. Continuous data collection on rare potential risks, and active surveillance of vulnerable populations, such as people with a proven history of allergic reactions, can help to identify the risks of PEG exposure and the development of increasingly effective and safe vaccines. This is particularly important when considering the extension of the use of PEGylated SLNP COVID-19 vaccines to the paediatric population.

The rapid production of the mRNA-based vaccines has been possible not only thanks to a huge effort in time, people and money, but also thanks to an enormous body of evidence accumulated in previous preclinical studies on similar nanocarriers aimed at nucleic acid delivery $^{25}$. Given their wide administration, one would therefore hope that the nano-based vaccines may 'return the favour' by providing robust indications about the safety and nanokinetics of SLNPs, leading to faster clinical translation of other nanotechnology-based biomedical products.

\section{P. Bigini1 ${ }^{1}$, M. Gobbi (D)1, M. Bonati',}

A. Clavenna', M. Zucchetti', S. Garattini ${ }^{1}$ and G. Pasut (iD) 2

${ }^{1}$ Istituto di Ricerche Farmacologiche Mario Negri IRCCS, Milan, Italy. ${ }^{2}$ Pharmaceutical and Pharmacological Sciences Department, University of Padova, Padua, Italy.

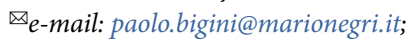
gianfranco.pasut@unipd.it

Published online: 4 November 2021 https://doi.org/10.1038/s41565-021-01001-3

\footnotetext{
References

1. CDC COVID-19 Response Team/FDA. MMWR. Morb. Mortal. Wkly Rep. 70, 46-51 (2021).

2. ACIP Presentation Slides: January 27, 2021 Meeting (ACIP, accessed 16 February 2021); https://www.cdc.gov/vaccines/acip/ meetings/slides-2021-1-27-21.html

3. McNeil, M. M. et al. J. Allergy Clin. Immunol. 137, 868-78 (2016).

4. de Vrieze, J. Science 371, 10-11 (2021)

5. Erman, M. Reuters (2020) https://www.reuters.com/article/ us-health-coronavirus-vaccines-fda-idUSKBN28T05J
} 
6. Zalipsky, S. \& Pasut, G. in Polymer-Protein Conjugates (eds Pasut, G. \& Zalipsky, S.) 3-22 (Elsevier, 2020).

7. Richter, A. W. \& Akerblom, E. Int. Arch. Allergy Appl. Immunol. 70, 124-31 (1983).

8. Sellaturay, P., Nasser, S. \& Ewan, P. J. Allergy Clin. Immunol. Pract. 9, 670-675 (2021).

9. Shimabukuro, T. T., Cole, M. \& Su, J. R. JAMA 325, 1101-1102 (2021).

10. Yang, Q. et al. Anal. Chem. 88, 11804-11812 (2016).

11. Fang, J.-L., Beland, F. A., Tang, Y. \& Roffler, S. R. Toxicol. Rep. 8, 148-154 (2021).

12. Lubich, C. et al. Pharm. Res. 33, 2239-49 (2016).

13. Armstrong, J. K. in PEGylated Protein Drugs: Basic Science and Clinical Applications (ed. Veronese, F. M.) 147-168 (Birkhäuser, 2009).
14. Khalil, A. et al. Haematologica https://doi.org/10.3324/ haematol.2020.258525 (2020).

15. Troelnikov, A. et al. J. Allergy Clin. Immunol. https://doi. org/10.1016/j.jaci.2021.04.032(2021)

16. Safra, T. et al. Ann. Oncol. 11, 1029-1033 (2000).

17. Neun, B., Barenholz, Y., Szebeni, J. \& Dobrovolskaia, M. Molecules 23, 1700 (2018).

18. Webb, L. M. \& Lieberman, P. Ann. Allergy Asthma Immunol. 97 39-43 (2006).

19. Peckham, H. et al. Nat. Commun. 11, 6317 (2020).

20. Serpooshan, V. et al. ACS Nano 12, 2253-2266 (2018).

21. Sellaturay, P., Nasser, S., Islam, S., Gurugama, P. \& Ewan, P. W. Clin. Exp. Allergy 51, 861-863 (2021).

22. Chisholm, C. F. et al. J. Pharm. Sci. 104, 3681-3690 (2015).

23. Krayukhina, E. et al. J. Pharm. Sci. 108, 2278-2287 (2019).
24. Selmin, F. et al. Pharmaceutics 13, 1029 (2021).

25. Hammond, S. M. et al. EMBO Mol. Med. 13, e13243 (2021).

Competing interests

G.P. has been scientific advisor for Takeda (Japan) and NOF (Japan), outside the submitted paper. The other authors declare no competing interests.

Additional information

Peer review information Nature Nanotechnology thanks the anonymous reviewers for their contribution to the peer review of this work. 